\title{
Efficient and Consistent Path loss Model for Mobile Network Simulation
}

\author{
Seon Yeong Han, Member, IEEE, Nael B. Abu-Ghazaleh, Senior Member, IEEE, \\ and Dongman Lee, Member, IEEE
}

\begin{abstract}
The accuracy of wireless network packet simulation critically depends on the quality of wireless channel models. Path loss is the stationary component of the channel model affected by the shadowing in the environment. Existing path loss models are inaccurate, require excessive measurement or computational overhead, and/or often cannot be made to represent a given environment. The paper contributes a flexible path loss model that uses a novel approach for spatially coherent interpolation from available nearby channels to allow accurate and efficient modeling of path loss. We show that the proposed model, called Double Regression (DR), generates a correlated space, allowing both the sender and the receiver to move without abrupt change in path loss. Combining DR with a traditional temporal fading model, such as Rayleigh fading, provides an accurate and efficient channel model that we integrate with the NS-2 simulator. We use measurements to validate the accuracy of the model for a number of scenarios. We also show that there is substantial impact on simulation behavior when path loss is modeled accurately. Finally, we show that unlike statistical models, DR can make a simulation representative of a given environment by using a small number of seeding measurements. Thus, DR provides a cost-effective alternative to ray tracing or detailed site surveys.
\end{abstract}

Index Terms-Wireless Channel Model, Mobile Network Simulation, Path Loss, Spatial Correlation

\section{INTRODUCTION}

Simulation is widely used for performance evaluation in wireless and mobile network research. The major advantages of simulation include flexibility in modeling scenarios, controllability of changing parameters that are difficult to change in practice, observability compared to testbeds or emulation studies, and repeatability. However, the validity of simulation models for wireless networks has been criticized due primarily to the poor accuracy of wireless channel models [1], [2], [3], [4], [5], [6], [7]. There are other aspects of wireless network simulation that have also come under criticism including the use of unrealistic mobility models [8], [9].

In response to this criticism, more accurate and realistic temporally fading channel models accounting dynamic components for signal strength due to mobility and multipath effect, e.g., Rayleigh-Ricean fading [10] and Nakagami fading [11], have been implemented in network simulators. However, these models account for the fast fading component of the channel model, which exhibits temporal correlation but little spatial correlation [12]. On the other hand, the stable component of

- S.-Y. Han is with Korea Electric Power Research Institute (KEPRI) as a postdoctoral researcher. Email: shan8017@gmail.com

- N. Abu-Ghazaleh is with the CSE and ECE Departments, University of California, Riverside.

Email:nael@cs.ucr.edu

- D. Lee is with the Department of Computer Science, Korea Advanced Institute of Science and Technology (KAIST).

Email: dlee@cs.kaist.ac.kr a signal is determined by shadows from large objects in an environment; this path loss component, is still commonly modeled assuming idealized distributions. However, path loss has been shown to be spatially correlated as RF shadows from large objects tend to have similar effect on nearby channels [13], [14]. The path loss component determines the mean signal strength and therefore significantly affects link quality [15]. Spatial correlation is extremely important for simulation accuracy especially in the presence of mobility: as a node moves at small scales, spatial correlation implies that the path loss correlates from one position to the next.

There are two major approaches to accurate path loss estimation for a given environment: (1) site surveys use extensive measurement to map the signal power from every location to every other location in a site [16]; and (2) Ray tracing: instead of measurement, signal propagation in a site is tracked in detail using geographical and material information specific to the site; the direct, reflected, refracted, diffracted, and scattered rays between a sender and a receiver are summed to estimate the average received signal strength for a specific location [17], [18], [19]. Both these approaches are accurate, but require significant measurement or computational overhead, which makes them unsuitable for use in a packet simulator.

One of the difficulties in producing spatially correlated path loss values in mobile-to-mobile network simulation is that both the sender and receiver could be mobile. As both the sender and the receiver move, it is not clear how to maintain spatial coherence for the successively generated channel models. In this paper, we propose 
a path-loss model for wireless network simulation capable of spatially coherent estimation of path loss in the presence of both sender and receiver mobility. The model maintains the spatial correlation of path loss which has been empirically observed in both outdoor and indoor scenarios [13], [14], [20]. Thus, the model produces consistently plausible path loss values: those that can possibly be observed in a real environment. To obtain a plausible path loss value for a new link, we use a new algorithm to estimate the channel from nearby links.When generating a new link, we search known nearby reference links. We then interpolate twice from these nearby links, once to account for the difference in receiver location and once for the sender; the algorithm is therefore called Double Regression (DR). For efficient identification of related reference links, we use a spatial data structure to index the nearby links. Although regression has been used to compute path loss in wireless simulation, existing techniques only allow one end of the channel to be mobile [21].

If there are insufficient reference data (known links that are sufficiently close) for regression, we generate the path loss value from a log-normal distribution. Finally, the newly generated link is stored as a known link for future estimation of nearby channels. As a result, unlike existing models, the path loss remains spatially coherent as well as temporally consistent: path loss values are preserved over time, mirroring the long-term nature of shadowing which determines the path loss.

We envision the model to be used in a way where a set of known channels are built over time initially starting from stochastic values, and then using interpolation as more channels are known. However, it is also possible to seed the model with measurement or ray-tracing channels creating simulations that are site specific. When seeded with known channel values, the model can "fill-the-gaps" with realistic values between the known measured ones, allowing faster site surveys or ray-tracing analysis where only a few channels are measured or ray-traced.

The model is validated against measured data using both new experiments, as well as large scale trace data. We integrate the path-loss with a temporal fading channel model (Rayleigh fading) to provide a complete channel model, and show that different path loss estimates cause substantially different link quality estimates. Even in a simple two-hop scenario, the use of spatially coherent models can result in significant difference in estimated throughput over existing models when there is only $10 \mathrm{dBm}$ difference in path loss. Finally, we show that seeding the simulation with some measurement data can substantially increase the accuracy of a site-specific simulation without the high cost of site surveys or ray tracing.

The remainder of the paper is organized as follows. Section 2 is an overview of some background and related work. In Section 3, we present the proposed double regression algorithm for spatially correlated path-loss estimation. We present our experimental evaluation of the channel model and the assessment of its impact on the accuracy of simulation studies in Section 4. Finally, Section 5 presents some concluding remarks.

\section{Background ANd Related Work}

Wireless channel models typically consist of two components that are summed to estimate the overall received signal strength. The first component is the long-term average signal strength called the path loss; DR is a path loss model that estimates this component of the channel. The second component represents the small-scale multipath fading to model the signal strength variation from multi-path effects as the signal propagates and interacts with the environment. This section overviews two commonly used combinations of these two components and discusses their limitations. We also review three advanced path loss models that are known to have high accuracy and compare them to DR.

\subsection{Commonly used Wireless Channel Models}

In network simulators, the following two model combinations are widely used: (1) Ideal path loss model with Rayleigh fading model (IPL-Rayleigh); and the (2) log-normal shadowing model with Rayleigh fading model (LNSM-Rayleigh). The ideal path loss model in the IPL-Rayleigh can be the free space model, the two-ray ground model, or any other isotropic distancedependent path loss model. IPL-Rayleigh is widely used because of its simplicity [10], [11]. However, a parameter of the fading channel model, the mean power, is obtained from ideal path loss models; thus, the mean power and therefore the average link quality of a link do not reflect a real site with characteristic shadowing effects.

The LNSM-Rayleigh is more realistic because the path loss is obtained by the log-normal shadowing model, which is widely accepted as a site-specific stochastic model [22]. However, the LNSM model assumes independent and identically distributed path loss for points at the same distance from a sender; it does not consider the spatial correlation between nearby points [13], [14]. Moreover, there is no effort to maintain temporal consistency for a channel (i.e., to make sure that if the channel is used later, it has the same parameters). Moreover, in some simulators LNSM is implemented by recomputing the log normal component with every packet transmission. Thus, even if no mobility occurs, the path loss component of the model changes substantially with every transmission.

In summary, the models used in current network simulators are highly inaccurate with respect to the path loss component of the model. In IPL, an idealized model is used for estimating path loss, essentially not capturing the effect of shadows in the environment. In contrast, LNSM uses a log normal component to estimate path loss, but does so in a way that is neither 
spatially nor temporally coherent. We show later that using these models can lead to simulation results that diverge significantly from both measurements and more accurate models.

\subsection{Advanced Path Loss Models}

In this section, we compare DR to other advanced path loss models. To set up the metrics for the comparison, we summarize the primary advantages of DR: (1) Spatial and temporal consistency: DR estimates path loss in a manner that is spatially coherent and temporally consistent; (2) Efficiency: DR is efficient both in terms of computation time, as well as the overhead needed to build the model; and (3) Flexibility: DR allows the integration of measurement results to model specific environments, or alternatively it allows populating the model statistically for channels where no nearby channels have been estimated before.

\subsubsection{Site Surveys}

One approach to providing a highly accurate path loss exponent is to use a site survey. A site survey is a process where signal power measurements are performed throughout an area which will be used as the basis for simulation. However, this process is extremely time consuming and expensive; often it is only feasible to perform measurements in a limited number of locations, and the quality of measurement depends on equipment and skill [16]. Due to these reasons, several methods to reduce the number of required measurements in planning of access points have been proposed [23], [24]. Stepanov et al use a commercial site survey tool (WinPROP) and present the significance of using an accurate propagation model in MANET study [25]. Compared to DR, SS can model specific environments, but does not have the flexibility to stochastically model new environments while maintaining consistency. It also has substantially higher set up overhead to carry out the detailed measurements.

\subsubsection{Ray Tracing (RT)}

In Ray Tracing, detailed geographical information about a site is collected and used as the basis of computing path loss. In particular, the direct, reflected, refracted, diffracted, and scattered rays between a sender and a receiver are summed to estimate the average received signal strength for a specific location [17], [18], [19]. RT is highly accurate if the precise geographic information (GIS) (e.g., materials, facets, and edges) about the environment, as well as the sender and receiver is available. However, when the typical number of facets and edges are used, the computational complexity easily gets into the trillions of rays [26]. This computational cost is prohibitive for packet simulation especially in the presence of mobility (since the path loss would have to be continuously re-estimated).

Dricot and Doncker implement the ray-tracing model in a slowly moving environment in a network simulator [27]. Because of the change of the environment, they re-calculated the ray-tracing result every $0.05 \mathrm{sec}$, which was assumed to be coherent time in the $2.4 \mathrm{GHz}$ channel. As a result, they noticed that the simulation runtime is increased 100 fold. Despite the efforts for increasing the efficiency of the ray-tracing, the computational complexity remains prohibitively high [28]. For example, one solution for this problem is to store the precomputed ray-tracing result for a whole city, if the location of the sender is fixed [28] (e.g., base station). However, this is not applicable to ad-hoc networks where both senders and receivers move. Due to computational inefficiency and the expensive cost of detailed GIS data, RT is not used as a path-loss model for packet simulators. Lu et al. propose a simplified ray-tracing model to calculate path loss [29]. They simplify the equation for path loss using an adjustable parameter $\mathrm{S}$ that describes the diffraction/scattering mechanism. Comparison with measurements is used to estimate the S. Since the parameter reflects the environment, the accuracy of their approach depends on the spatial resolution of measurements, and error bounds are not known.

\subsubsection{Sum-of-Sinusoids model}

The Sum-of-Sinusoids model was initially developed by Rice [30] and advanced and extended by others [31], [32], [33], [34]. For two arbitrary links, the autocorrelation is determined by the distance between the senders and the receivers as each link's path-loss is a Gaussian random variable. Autocorrelation is achieved by selecting the frequency set defined in the SOS formula carefully. Like $\mathrm{DR}$, SOS can generate a spatially correlated log-normal shadowing model and is computationally efficient. However, unlike DR, SOS is completely stochastic and cannot be used to create site specific models due to lack of location information of nodes.

\subsubsection{Correlated Shadowing model}

Correlated shadowing is a model proposed by Szyszkowicz et al. [35]. In this model, a fast shadowing field generation algorithm is proposed that takes the angle and distance of arriving signal. The method takes $N$ points for receiver's location and constructs a pairwise covariant matrix. For an unknown location, they round to the nearest quantization point. Thus, path loss changes abruptly in the boundary of the given data locations. Moreover, the work is limited to a cellular system that consists of base stations and mobile nodes; it does not apply to channels where both sender and receiver move as encountered in mobile ad hoc networks.

Also related to our work, $\mathrm{Xu}$ et al [21] use regression to estimate link quality to add spatial coherence to temporal correlation. They do not consider separate regression from the source and destination side. Moreover, they attempt to estimate the full link quality, rather than just the stationary spatially coherent component representing path loss. In particular, a sliding window of recently perceived link qualities are maintained and as 
time passes, the quality of the link can drift by arbitrary amounts.

\subsubsection{Empirical Path Loss Models}

In addition to the free space model and the two-ray ground model, several empirical channel models, such as the Okumura model, the Hata model, and the WalfishIkegami model, have been developed for outdoor scenarios [36]. In particular, the Hata model and the WalfishIkegami model are used with a standard set of coefficient values such as building height, street width, building separation, and base station antenna height [37]. These outdoor scenario channel models are not applicable to indoor scenarios or local area networks because they usually assume the height of the antennas to be tens of meters and the transmission power to be strong enough to have a much larger transmission range.

When the height of antenna is low, the path loss is dominated by various near surface structures and vegetation. Azevedo and Joaquim compare several nearground path loss models designed for locations with vegetation such as COST 235 and FITU-R with lognormal shadowing model [38]. Those vegetation path loss models require the depth of vegetation area to estimate the path loss. They are not suitable for urban areas or indoors.

\section{Double Regression: Spatially coher- ENT Estimation Of PATH loss}

Our goal in this paper is to develop a plausible model for path loss: the long term stable component of a wireless channel model. Plausibility for path loss entails maintaining spatial and temporal consistency across the simulation lifetime. Spatial consistency implies that spatial correlation is maintained such that nearby channels have correlated path loss at correlation levels consistent with those measured empirically. If there are no neighboring links, neighbor relationship being defined using empirically observed correlation distance thresholds, the estimated path loss can be independent; for example, it can be generated by the log-normal distribution. However, as links are generated they populate the model, and eventually there are bases for estimation available for all new channels. Since these links are stored, they also provide the basis for temporal consistency such that similar channels used at different simulation times are also correlated.

This section overviews DR. We follow the notation used in log-normal shadowing description by Rappaport [12]: i.e., $X_{\sigma}\left(t_{x}, t_{y}, r_{x}, r_{y}\right)$ is a normally distributed random variable $N\left(0, \sigma^{2}\right)$ that models the path-loss component of a link $t(x, y) \rightarrow r(x, y)$. The path loss component represents the long term average signal power on a channel (i.e., not considering the zero mean multi-path fading component).

\subsection{Path Loss Estimation using Double Regression}

Regression is a process of least square fitting used to estimate a dependent variable from one or more independent variables [39]. Without loss of generality, we use linear regression (Eq. 1) due to its low computational complexity.

$$
p=\beta_{0}+\beta_{1} x+\beta_{2} y,
$$

where $x$ and $y$ are the independent input variables and $p$ is the computed dependent value. Given a set of $m$ observations $\mathbf{O}=\left\{\left(x_{j}, y_{j}, p_{j}\right) \mid j=1,2, . ., m\right\}$, the regression coefficient can be obtained by a weighted average of the different channels with weights inversely proportional to their distance. More specifically,

$$
\beta=\left(\Phi^{T} \Phi\right)^{-1} \Phi^{T} \mathbf{p}
$$

where $\mathbf{p}=\left(p_{1}, p_{2}, . ., p_{m}\right)^{T}$ and $\Phi$ is a $m \times k$ matrix of $\left[\Phi_{i}\left(x_{j}, y_{j}\right)\right], i=0,1, . ., k$ and $j=1,2, . ., m$.

The primary difficulty in using regression is that the notion of distance in regression is a scalar value reflecting Euclidean distance between the reference and projected points. In contrast, channels are line segments in space with no clear definition for distance between them. Our approach, Double Regression (DR), solves this problem by applying regression in two steps. First, we apply regression based on the distance from the destination of the nearby links to the destination of the estimated link producing an intermediate estimate. In the second step, we apply regression based on the sender distances to the intermediate estimate to find the final path loss. The remainder of this subsection formalizes this process.

For ease of explanation, we describe DR in a twodimensional plane, but it readily generalizes to three dimensional space. Consider a path loss offset $X(x, y, u, v)$ of a link $(x, y) \rightarrow(u, v)$ where $(x, y)$ and $(u, v)$ represent the location of a sender and a receiver respectively. Let the location coordinates $x, y, u, v$ be the independent variables of the regression and the $X$ be the dependent variable. When a set of neighboring links are given (from previous estimates, measurement, or pre-calculated ray tracing), the path loss offset $(X(x, y, u, v))$ for a new link needs to be estimated. To distinguish neighboring links from those not sufficiently close to be correlated, we define a correlation distance threshold, $D_{n}$, beyond which we do not consider links to be related. If $(x, y)$ is static, and $m$ observations for $\left(u_{1}, v_{1}\right), . .,\left(u_{m}, v_{m}\right)$ are given within $D_{n}$, regression esimates an offset for a new link $(x, y) \rightarrow\left(u^{\prime}, v^{\prime}\right)$. The underlying assumption of the regression is that the relationship between the independent variables (i.e., locations) and their dependent variables (i.e., the path-loss) among the observations continues to hold between the new independent variable $\left(u^{\prime}, v^{\prime}\right)$ and its dependent variable $X\left(x, y, u^{\prime}, v^{\prime}\right)$. The assumption relies on the dependent variables having spatial correlation, consistent with empirical data [13], [14], [20]. 


\subsection{DR Algorithm}

To lower the overhead of finding the reference links for regression, we use an R-tree, a data structure that supports efficient spatial indexing [40]. A leaf node of the Rtree contains the location of the sender, the receiver and the observed path loss. Figure 1 and 2 show a geographical illustration of an R-tree that covers 7 links (14 links considering the reverse links), and the corresponding Rtree data structure. A branch node represents a region and a leaf-node represents the sender's location of a link. A new leaf node is created such that that the sender's location is within the region of the parent branch node. Since a path loss is symmetric [12], a link with opposite direction and the same path-loss value is also stored in the R-tree. R1 covers all links while R4 covers only link $\mathrm{b} 1 \leftrightarrow \mathrm{b} 2, \mathrm{a} 1 \rightarrow \mathrm{a} 2$, and $\mathrm{c} 1 \rightarrow \mathrm{c} 2$. Each directed link between two nodes is stored in a separate R-tree node and the R-tree node is assigned depending on the sender's location. When a new estimation is requested, reference links within a correlation distance threshold $\left(D_{n}\right)$, up to a limited number $(L)$ can be obtained quickly by traversing the R-tree. If the number of reference neighbor links is not limited, the computation time can increase as the number of measurements increases. The distance between two links $(x, y) \rightarrow(u, v)$ and $\left(x^{\prime}, y^{\prime}\right) \rightarrow\left(u^{\prime}, v^{\prime}\right)$ is calculated as follows.

$$
\begin{aligned}
d t & =\sqrt{\left(x-x^{\prime}\right)^{2}+\left(y-y^{\prime}\right)^{2}} \\
d r & =\sqrt{\left(u-u^{\prime}\right)^{2}+\left(v-v^{\prime}\right)^{2}} \\
d i s t & =\sqrt{d t^{2}+d r^{2}}
\end{aligned}
$$

where $d t$ represents the distance between transmitters and $d r$ represents the distance between receivers.

The estimation algorithm operates as follows. Let us consider a set of reference links $\mathbf{R L}$ and a link $\tau$ that has a sender $A$ and a receiver $B$. We denote each coordinate of a node by $i d(e)$, where $e=x, y, u$, or $v$. Given a set of reference links, DR finds a set of regression coefficients that fits the path loss offset $X$ at receiver locations of reference links, assuming for now the same transmitter location (line $1 \sim 9$ in the DR algorithm in the right column). The offset $X$ at the receiver location of interest is estimated with the obtained regression coefficient sets, resulting in a set of pre-estimates (line 10). The above steps are repeated for each reference transmitter location (line 11). A set of regression coefficients is next fitted to these pre-estimates across the reference transmitter locations (line 12 13). In the final step, the path loss offset of the link of interest is obtained by evaluating the regression function fit at the desired transmitter location (line 14).

The detailed description of the algorithm with the aforementioned notation is presented below. The algorithm applies regression twice, one for receivers and one for senders. This method enables estimating any link, including those whose sender and receiver are different from end nodes of reference links. If there are no available reference links, we use a stochastic estimate

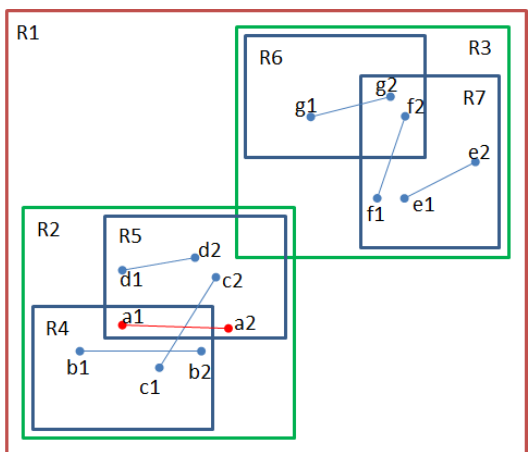

Fig. 1: R-tree coverage

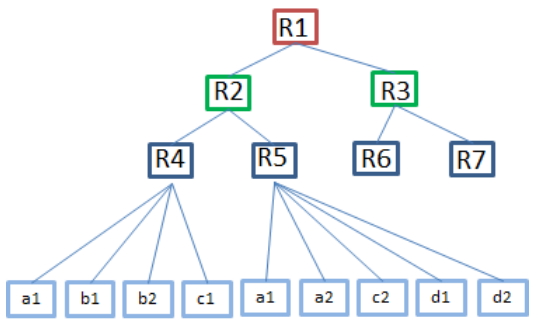

Fig. 2: R-tree

(log-normal distribution) to compute the path loss.

Double regression algorithm

$1 I=$ the number of reference links in $\mathbf{R L}$

2 for $i=1$ to $I$ do

$3 \quad s_{i}=$ the sender of $i^{\text {th }}$ reference link

$4 \quad J=$ the number of receivers for $s_{i}$

$5 \quad r_{i j}=j^{t h}$ receiver of node $s_{i}$

// observation set for a sender $s_{i}$

$6 \quad \mathbf{O}_{s_{i}}=\left\{\left(r_{i j}(u), r_{i j}(v), X\left(s_{i}, r_{i j}\right)\right) \mid j=1,2, . ., J\right\}$

$7 \quad$ for $j=1$ to $J$ do

$8 \quad$ Apply Eq( 2) to $\mathbf{O}_{s_{i}}$ to get $\beta$ s.

9 end for

// get the estimated value for the target receiver location $B$ 10 11 end for Apply $\mathrm{Eq}(1)$ to $B$ and get the estimation $\hat{X}_{s}\left(s_{i}, B\right)$.

// observation set consisting of estimation for links to B

$12 \mathbf{O}_{s}=\left\{\left(s_{i}(x), s_{i}(y), \hat{X}\left(s_{i}, B\right)\right) \mid i=1,2,,, . I\right\}$

13 Apply Eq( 2) to $\mathbf{O}_{s}$ to get $\beta$

14 Apply Eq( 1$)$ to $A$ and get the estimated value $\hat{X}(A, B)$

\subsection{Tuning the R-tree to balance accuracy and over- head}

A major challenge in using R-tree in path loss model is that the data in the original $\mathrm{R}$-tree represent 2dimensional points while ours represent links consisting of two end positions. Simply increasing the dimension is not appropriate, because a branch node covering the long links will be very large. An R-tree consisting of branch nodes covering a large area is usually computationally inefficient, because the branch will have a lot of leaf nodes or the coverage of neighbor branch nodes will be mostly overlapped if the number of leaf nodes is limited. To avoid this problem, the R-tree branch nodes in our model are constructed based on the sender position. Thus, the receiver position is used to check 
whether the link could be a reference once the candidate links are found. Since we inserted each link twice to reflect the reverse channel, considering only senders in practice indexes both senders and receivers. Note that the other end of the link in a branch node can be outside of the coverage of the node making them inappropriate for use as reference links. Thus, the candidate links found in an area must be checked to see if both ends fall within the spatial coherence distance $D_{n}$.

We use two parameters to control the use and construction of the R-tree to tradeoff accuracy to computational and storage overhead: $T h_{\text {Cover }}$ and $T h_{\text {Save }}$. To select a set of reference links for a link $a 1 \rightarrow a 2$, R3 does not need to be searched, which leads to faster search time. Since a link is covered by multiple layers of regions (e.g., link b1 $\rightarrow$ b2 is covered by R1, R2, and R4), we have to determine which region is appropriate, i.e., including all related links and excluding unrelated links. Th $h_{\text {Cover }}$ controls the search region for related links within the tree measured in meters. A small $T h_{\text {Cover }}$ means that the search progresses to deep levels of the tree until a region is found that covers the target area tightly due to the small $T h_{\text {Cover }}$. In this case, a small number of neighboring links are considered as candidate references for estimating the new link. A shortcoming of this approach is that a close neighboring link may be excluded because its end points are out of the covered area. For example, the closest link to link $\mathrm{a} 1 \rightarrow \mathrm{a} 2$ is $\mathrm{b} 1 \rightarrow \mathrm{b} 2$, however, if $\mathrm{R} 5$ is selected, the closest link will not be found as a reference. If a large $T h_{\text {Cover }}$ is used, the search stops higher in the tree at a larger region, which leads to more links being considered for reference links. Thus, the probability that a close neighbor link is excluded from a reference set of a link is low, but a higher number of links will be considered.

The DR model stores an estimated path loss in the Rtree to reuse. The interval between two adjacent stored links can be adjusted using $T h_{\text {Save }}$. If a small $T h_{\text {Save }}$ is used, we allow a dense R-tree to be constructed where many nearby links are saved. This choice requires more storage space, and adds to the overhead of a new channel estimate, but allows more accurate estimates. Conversely, if a large $T h_{\text {Save }}$ is used, less reference links are used, saving storage and computation time, but increasing the stochastic component of the estimate. If a link is estimated without any reference link, the model stores the estimated value.

\subsection{Discussion}

DR applies regression when nearby known channels are available. These channels could be obtained from measurement data to produce a site specific simulation. In such scenarios, the accuracy of the DR estimate relative to the site from which the measurement data is obtained depends on the presence of nearby measured (or ray-traced) links; absent such links, there is no spatial correlation from measured links and the estimated path loss is independent in the initial phase of the simulation where the R-tree is sparsely populated.

DR always generates plausible path loss models even when the provided measurement or ray-trace data is sparse or even non-existent; the generated path loss values will be consistent with path loss distribution in real environments and are thus representative of a hypothetical real environment. However, if the measurement (or ray-trace) data is sufficiently dense, DR will generate site-specific path loss representative of the actual environment from which the measurement data are obtained. The measurement or ray-tracing data simply causes the generated path loss values to correlate to this data making the simulated area similar to the measured area.

\section{Validation and Performance Evalua- TION}

In this section, we present a number of experiments to validate DR and to evaluate its properties. We also show study the impact of having plausible path loss models on network performance and stability.

\subsection{Measurement-based Validation of DR}

In this study, we measure the path loss in a lecture hall that has dimensions of $10 \mathrm{~m}$ by $10 \mathrm{~m}$ by $3 \mathrm{~m}$. We carry out measurements from six sender locations. For this experiment, the room structure such as walls, furniture and ceiling affects the path loss. The sender broadcasts packets every $10 \mathrm{msec}$ with transmission power of $16 \mathrm{dBm}, 802.11 \mathrm{~g}$ in $2.471 \mathrm{GHz}$ frequency, and $6 \mathrm{Mbps}$ data rate. To eliminate multi-path effect (the temporal fading component of the signal) and orientation effect [41], [42], the signal powers are averaged in a small area of $1.8 \mathrm{~m}$ rotating the receiver antenna in all directions; in total, 147 channels are measured. Both the sender and the receiver use Atheros chipset AR5112 and the MadWifi driver set for linux 2.6.

Given the measurement data, we seek to do parameter estimation for the log-normal model. Since the lognormal model is used as the stochastic component of DR, this step is important not just for comparison against lognormal shadowing. Given a measured channel $S\left(t_{i}, r_{i}\right)$, we first seek to separate the log-normal component of the path loss from the stable component which is the average path loss. This process requires us to estimate the $P_{0}$ parameter of the log normal model which represents the power at the cross over distance $d_{0}$ and the path loss exponent, $\beta$, to get the average path loss [12]. Note that $\beta$ can be fixed for a site because the variable path loss is represented by the variable component of the log-normal model.

We also need to estimate $\sigma$ which is the parameter of the log-normal distribution component of the shadowing 
model. First, the power $P_{d b}$ is obtained using $S-95$ per the specifications of our hardware [43]. To obtain $P_{0}$ from the measurement, we set $d_{0}$ to be 1 . To obtain $\sigma$, we need to determine $\beta$ first: $\beta$ is computed such that it results in a zero mean for the log-normal distribution which minimizes $\sigma$. In our testbed, the values $P_{0}=-33 d B, \beta=$ $2.87, \sigma=4.06$ result from this exercise.

The simulated path loss values are compared with the measurement of the corresponding physical location as well as path loss values generated by the two ray ground model and log-normal shadowing model with the derived parameters described above. To make the average of the path loss generated by those models the same with the measured value, the $\mathrm{Gt}^{*} \mathrm{Gr}$ parameter is adjusted, which models the antenna gains of the sender and the receiver, of the two ray ground model (1.062 for this site) and used the derived $P_{0}, \beta$ and $\sigma$ in the testbed for $\log$ normal shadowing model. For the DR experiment, we use DR to interpolate each link from a preset number of neighboring measurements.

To assess the similarity, we calculate the root mean square (RMS) of the estimation error defined as $x_{R M S}=$ $\sqrt{\frac{1}{n} \sum_{i=1}^{n}\left(x_{i}-m_{i}\right)^{2}}$, where $n$ is the number of measured links, $x_{i}$ is the estimated pathloss in $\mathrm{dB}$ from the model for the $i^{t h}$ link, and $m_{i}$ is the measured pathloss in $\mathrm{dB}$ for the same link. RMS was computed across all measured links in the experiment, simulation: RMS was 9.96 for the two ray ground model while log-normal shadowing had an RMS of 12.843. DR achieves an RMS of 5.03. The reason is that the model estimates signal strength at each location from a few reference links that are obtained through measurement, while the two ray ground model estimates them with distance and log-normal shadowing model estimates them with only overall statistics and is not able to incorporate measurement values.

Site Surveys require a large number of measurements. Similarly Ray Tracing requires many computationally intensive computations. In contrast, DR can successfully fit an environment with only a few number of measurements, providing a geographically consistent model at a significantly lower cost than site surveys and Ray Tracing. Table 1 shows the RMS of estimation errors for various number of reference links used. We use R/M to indicate the ratio of samples used in seeding DR to the total number of measurements available (which is 147 in this experiment). When zero $\mathrm{R} / \mathrm{M}$ is used, the RMS value of $\mathrm{DR}$ is no larger than the log normal shadowing model because the same site specific statistics are used. As the R/M increases, DR generates more accurate path loss. Thus, the site fidelity can be adjusted by varying $\mathrm{R} / \mathrm{M}$.

In this next experiment, we use a measurement data set from the CRAWDAD repository measuring path loss in part of the University of Colorado campus [44] for outdoor scenarios. The data contains received signal strength (RSS) measurements collected using the CU

\begin{tabular}{|l|l|l|l|l|l|l|}
\hline \multicolumn{4}{|l|}{ two ray ground } & \multicolumn{4}{l|}{9.961} \\
\hline \multicolumn{4}{|l|}{ log-normal } & 12.843 & \multicolumn{4}{l|}{} \\
\hline \multirow{2}{*}{ DR } & R/M & $0 \%$ & $10 \%$ & $30 \%$ & $50 \%$ & $70 \%$ \\
\cline { 2 - 7 } & $x_{R M S}$ & 10.67 & 9.40 & 6.78 & 5.03 & 4.52 \\
\hline
\end{tabular}

TABLE 1: RMS of Estimation Errors

Wide Area Radio Testbed (CU-WART). Figure 3a shows a heat-map of the measured RSS. The total number of used links is approximately 900. The areas where no measurements were taken are shown as white. To show that DR can regenerate an accurate RSS map from a small number of measurement data, we reproduce a heat-map of RSS with 1/10 sample measurement (i.e., 90 samples) that are randomly selected.

As shown in Figure 3b, the log-normal shadowing model does not generate a realistic path loss model, because it uses statistics that do not reflect geographic characteristics such as low path loss in corridors. In the case of DR, since it is seeded by the reference measurement data the obtained path loss is similar to the measurement data as shown in Figure 3c. The computed RMS was 13.35 and 6.03 for the log-normal shadowing model and DR respectively.

\subsection{DR without Measurement: Correlated Gaussian Process}

DR can also generate a plausible path loss when no measurement data is available to seed the model. In this respect, the generated model is plausible, but not specific to any given area since it is not seeded with initial measurement data. In this way, the model can be used to evaluate network protocols given realistic path loss properties, without the overhead of any measurements or ray-tracing.

First, a distance threshold $\left(D_{n}\right)$ can be set to a decorrelation distance [33] because points at this distance away from each other experience weak correlation in path loss. In this case, the R-tree is originally empty. We can generate some initial reference links (e.g., on a grid $D_{n}$ meters apart) to seed the R-tree, or seed it on demand whenever a new link is needed and no nearby reference links exist. When no nearby links exist for a new channel, a Gaussian random variable from $N\left(0, \sigma^{2}\right)$ is used to assign the path loss at that point. The new estimate is added to the R-tree such that future nearby channels can use it in generating their estimate.

Figure 4 depicts the virtual signal-strength space obtained from double regression, where the sender is fixed at the middle of the topology and the $(r x, r y)$ coordinate represents the location of a receiver. The number of reference neighbors is set to 3 , and the distance threshold $D_{n}$ is set to 6 meters that is typical in an urban area [33]. We first generate channels at grid points $D_{n}$ apart; those points are generated independently because of the lack 


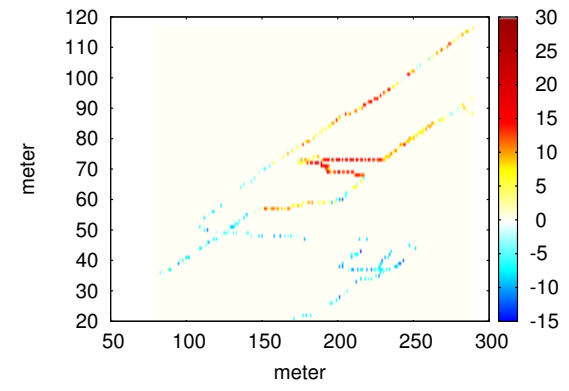

(a) Measurement RSS

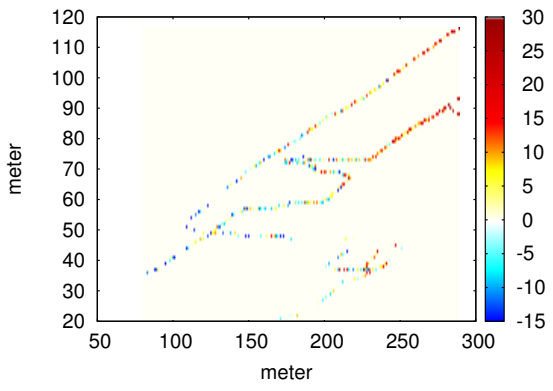

(b) Log-normal Shadowing RSS

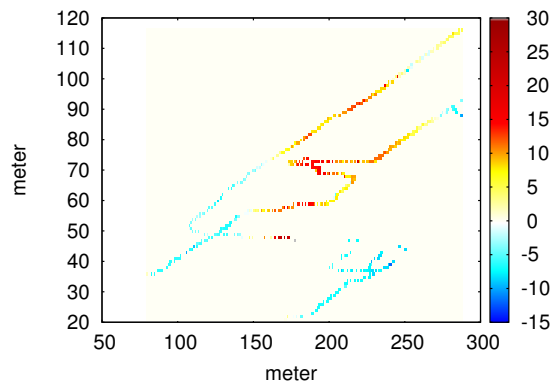

(c) DR Estimated RSS

Fig. 3: Estimated RSS using DR for the CU Wireless Trace

of nearby reference links. It is not necessary to seed the model with these points, as channels could be generated strictly on demand. The spatial correlation among the receivers, i.e., the similarity among the neighbor receivers, is plotted. We obtained similar results when a receiver is fixed and the $(x, y)$ coordinate represents the location of a sender. As a result, a small location change for either senders or receivers does not cause abrupt change of path loss, and the new link has a plausible path loss consistent with empirical results that show spatial correlation of path loss. While the spatial variation changes smoothly in most areas, it changes abruptly at the grid points which are generated without correlation (using a log-normal distribution).

Figure 5 compares the cumulative distribution function (CDF) of the offset $X(x, y, u, v)$ of the overall space to that at the grid points and CDF of log-normal distribution with variance of 1 and $\frac{1}{3}$ respectively.

The distribution of grid points shows 3 times larger variance than the distribution of overall space since they were generated independently. Averaging $n$ random variables for the target receiver and sender results in a reduced variance by $\sqrt{n} \times \sqrt{n}$ by the central limit theorem. Since we use an average of 3 reference links, the reduction in variance relative to the grid points follows the expected distribution.

Finally, Figure $6 \mathrm{a}$ to $6 \mathrm{c}$ show the joint autocorrelation with respect to $d t$ and $d r$. The DR generated path loss shows a good agreement with theory as does the SOS model [33]. The simulation result indicates that the DR model is well fit to the spatial correlation and smoothly changing path loss for mobile senders and receivers.

\subsection{Spatial Coherence Analysis}

In this section, we analyze DR with respect to the spatial correlation among the generated path loss estimates. Primarily, when no nearby links are available for regression, DR uses an uncorrelated Gaussian estimate for path loss. The density of the observations is varied by changing the correlation distance threshold $\left(D_{n}\right)$, which defines the distance beyond which links are no longer considered neighbors. When $D_{n}$ is large, simulating dense measurements, more neighbors will be included in the DR estimate. When it is small, simulating sparse measurements, many points will have no measured neighbors and would have to be generated using the Gaussian distribution, increasing randomness.

Figure 7 and 8 show autocorrelation when the receiver distance $(\mathrm{dr})$ and transmitter distance $(\mathrm{dt})$ between the reference and estimated link increases. When the distance between links is small (e.g., 1), the autocorrelation should be high (closer to 1). A lower autocorrelation indicates that the estimated path loss is not correlated in space. The topology size is $400 \times 400$, and 2000 reference observations are generated from $N\left(0, \sigma^{2}\right)$. When $D_{n}=30$ is used, explicit exponential autocorrelation is shown, which means if there are sufficient neighbor links, DR generates spatially correlated path loss. However, due to the random variables involved in the regression process when distance from reference links is large or insufficient nearby reference links exist, the autocorrelation does not look smooth. Each point represents an average of 720 simulation runs.

We vary the number of reference links in the same size of topology. If fewer references are used, more links are generated independently because they do not have sufficient neighbors for interpolation. Figure 9 shows that the autocorrelation is not seriously affected when the number of initial channels is reduced to 300 .

Figure 10 shows the autocorrelation between two links that are 1 and 5 meters apart as the number of reference links in the simulation increases. At first, there is no reference link $(\mathrm{x}=0)$. As a new link appears and an estimation for the link is stored in the R-tree, there will be more data that can be referred by a new link. When the number of links is small, the autocorrelation is low. Figure 10 shows that when the number of links reaches $2000(x=2000)$, the autocorrelation converges to match the empirical values. This simulation result can be also used to determine the number of initial links to generate a realistic path loss model. Each point represents the average of 30 simulation runs.

\subsection{Impact on Network Stability}




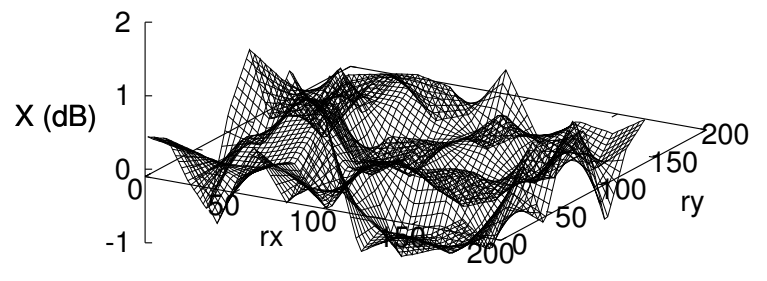

Fig. 4: A Realization of Path Loss Using DR

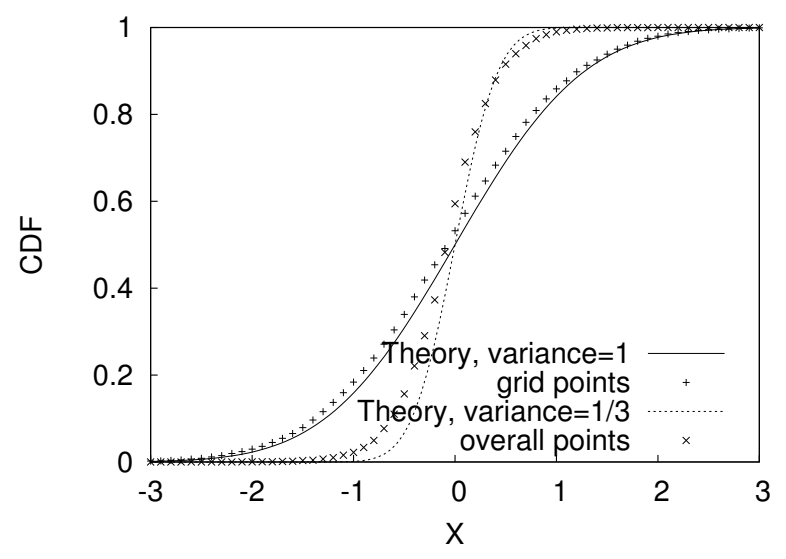

Fig. 5: CDF of double regression

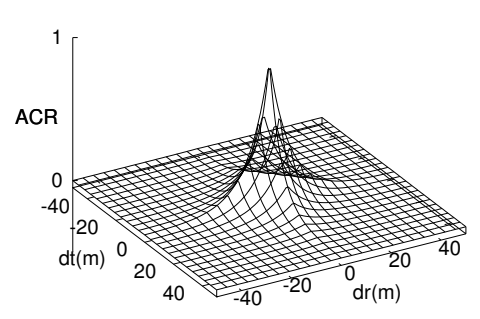

(a) TheoryACF

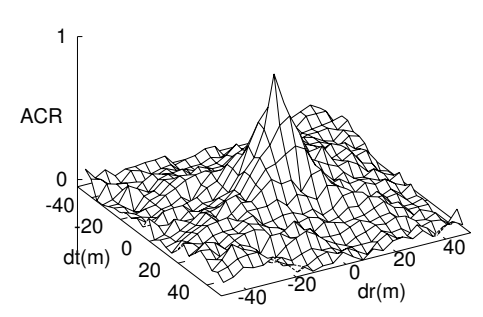

(b) SOS method

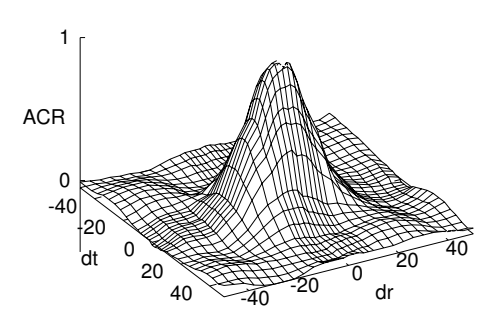

(c) DR

Fig. 6: Correlation Comparison

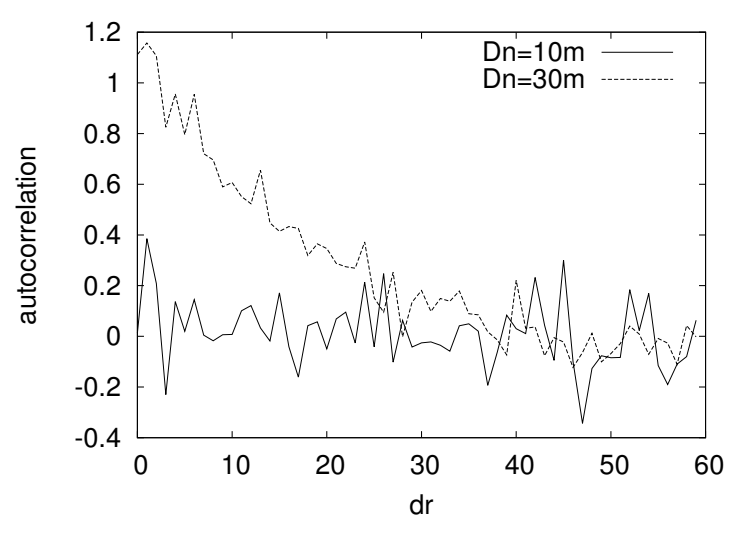

Fig. 7: Autocorrelation with receiver distance

The path loss models the long time average of the signal power; it has substantial impact on the simulation results, affecting the signal and interference components of SINR and how they change as the nodes move. This impact percolates up the protocol stack to the routing and application layers; the use of inaccurate path loss models can lead to substantial inaccuracy in the simulation. To demonstrate this effect, in this section, we evaluate the impact of path loss models on the behavior of two representative simulation studies.

One of the most notable impacts of the spatial corre-

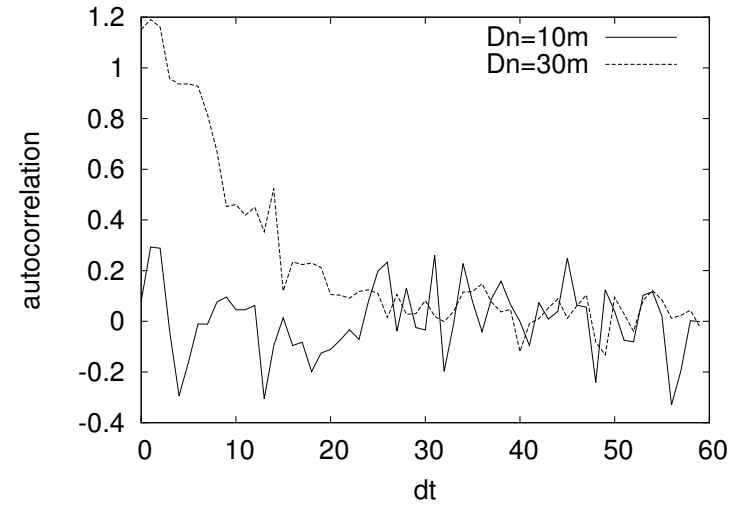

Fig. 8: Autocorrelation with transmitter distance

lation is on network stability. A representative reactive routing protocol for a mobile ad-hoc network, Dynamic Source Routing (DSR), detects a route failure after consecutive transmission failures, then re-issues the DSR route discovery process [45]. As mobile nodes move, the path loss and the link qualities between nodes keep changing, which causes route failure and rediscovery process.

We investigate how many times the re-discovery process occurs in the independent and identically distributed (i.i.d.) log-normal shadowing path loss model 


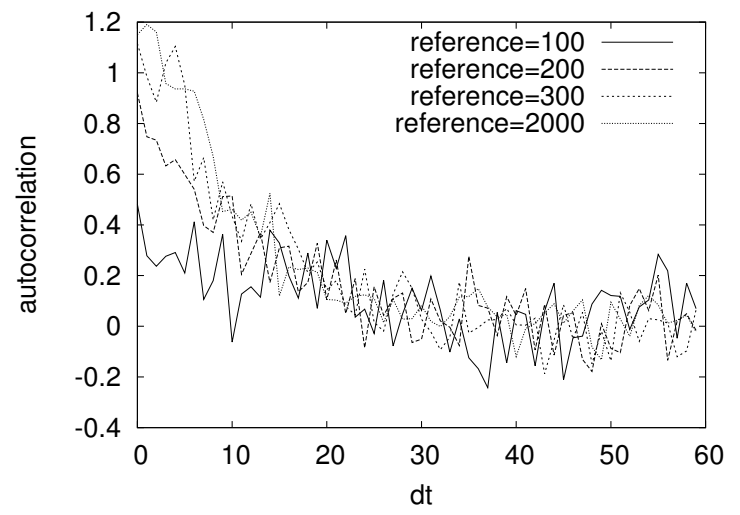

Fig. 9: Impact of the number of Initial Measured F Links

(commonly used in network simulators) and the DR path loss model in a general mobile ad-hoc network scenario. The scenario includes 20 nodes in a $30 \times 30 \mathrm{~m}$ topology in which each node moves at maximum speed of $1 \mathrm{~m} / \mathrm{sec}$. To make sure that the network includes connection and disconnection through multi-hops, the path loss exponent is set to 5 . Since the nodes move, a Rayleigh fading channel is used in addition to the path loss model to account for multi-path fading.

Fig. 11 shows the number of route discovery DSR packets normalized by the number of data packets. When the DR path loss model is used, the link quality of a pair of mobile nodes will change slowly, because the path loss is highly correlated with the distance. Thus, a small change of location probably does not cause a route failure. However, i.i.d shadowing path loss model does not guarantee the consistent link quality as nodes move even slowly, because the path loss for different location is determined independently. As a result, the i.i.d shadowing path loss model overestimates route failures and re-discovery processes as the standard deviation increases, while the DR model shows comparatively stable behavior.

A less stable routing layer will reduce the throughput and increase latency and jitter, because the time and channel capacity are wasted by the routing overhead. Our investigation of the impact on the throughput showed that using i.i.d shadowing channel model will underestimate the throughput by up to $42 \%$. This result highlights the significance of using a correct channel model in a simulation-based research.

\subsection{Impact on Throughput}

The next study shows the impact of the realistic pathloss model on throughput when we combine the model with a temporally fading model (Rayleigh fading). The symbol loss rate in a Rayleigh fading channel is:

$$
1-e^{-\rho^{2}} \text {, }
$$

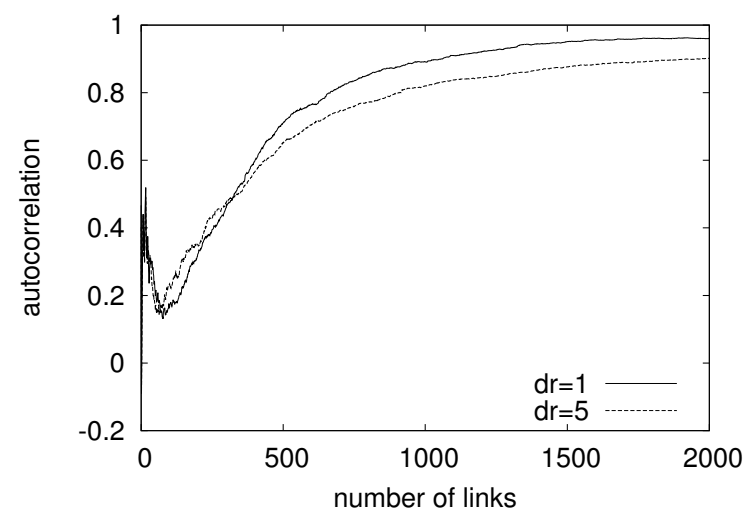

Fig. 10: Path loss stability as the number of generated links increases

where $\rho=\frac{R_{\text {threshold }}}{R_{R M S}}$ [15]. The symbol loss rate is an indicator of a link quality used in Rayleigh fading model that multiplies the duration the signal spends below the threshold and the rate of change of the fading. The symbol loss rate (which affects the packet loss rate) is determined by path loss, mobility speed, carrier frequency and $R_{\text {threshold }}$. Figure 13 depicts the delivery ratio of packet size of 30,512, and 1024 bytes. The $\mathrm{x}$-axis represents the attenuated signal strength due to path loss, $R_{R M S}=\sqrt{10^{(A(d)-30) / 10}}$, and the threshold $R_{\text {threshold }}=\sqrt{10^{(-90 d B m-30) / 10}}$. Since the signal strength under the Rayleigh fading channel fluctuates over time, the packet delivery ratio (PDR) increases gradually around the threshold as the attenuated signal strength due to path loss increases. Note that small differences in path-loss can result in large differences in PDR. For example, for packet size 1024, when the attenuated signal is incorrectly estimated by $10 \mathrm{dBm}$ (e.g., let's assume $-50 \mathrm{dBm},-40 \mathrm{dBm}$,or $-30 \mathrm{dBm}$ are used instead of $-60 \mathrm{dBm},-50 \mathrm{dBm}$, or $-40 \mathrm{dBm}$ ), the PDR changes from 0 , 0.4 , or 0.8 to $0.4,0.8$, or 1.0 , respectively.

The different PDRs cause significant difference in throughput especially over a multi-hop connection. We consider a two-hop connection A-B-C. We keep the PDR of A-B at a relatively high (0.83) and investigate the throughput as the PDR of B-C varies. Figure 14 depicts the impact of the various PDR on the throughput when various fading levels $\left(f_{m}\right)$ of the Rayleigh fading channel are used for each hop. If the aformentioned PDRs that are estimated with incorrect attenuation estimations (i.e., $0.4,0.8$, and 1.0 instead of $0,0.4$, and 0.8 ) are used for the second hop, the throughput are $25 \mathrm{kbps}, 150 \mathrm{kbps}$, and $350 \mathrm{kbps}$ instead of $0 \mathrm{kbps}, 25 \mathrm{kbps}$ and $150 \mathrm{kbps}$ when the second hop's $\mathrm{fm}=12 \mathrm{~Hz}$. The throughput difference are $25 \mathrm{kbps}, 125 \mathrm{kbps}$, and $200 \mathrm{kbps}$. This result supports the importance of estimating path-loss accurately in a multihop connection. 


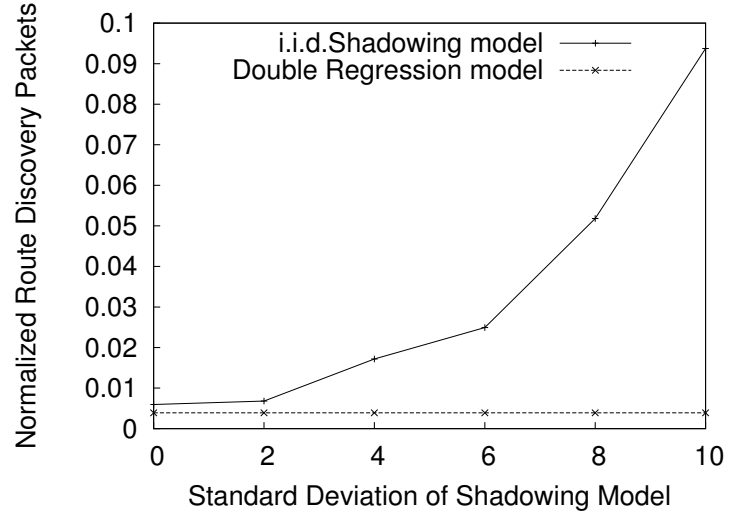

Fig. 11: Impact of spatial correlation on routing stability

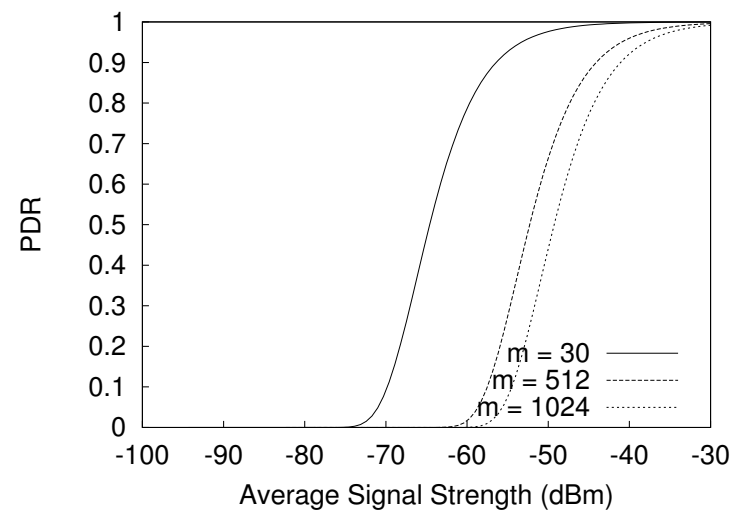

Fig. 13: PDR: Rayleigh fading and different packet sizes

\subsection{Impact on Model Performance and Accuracy}

Since a larger $T h_{\text {Cover }}$ allows more neighboring links for estimation, the accuracy of the model increases and the RMS decreases as $T h_{\text {Cover }}$ increases (Figure 15). However, if $\mathrm{R} / \mathrm{M}$ is small, the R-tree is scarcely populated which results in a limited number of neighboring links even as $T h_{\text {Cover }}$ increases. In Figure 15, when R/M=0.1, $T h_{\text {Cover }}=20$ shows the best RMS. This means an optimal $T h_{\text {Cover }}$ can be determined by the R/M.

A high $T h_{\text {Cover }}$ increases computational delay because a larger number of candidate neighboring links are considered. Figure 16 shows the computational delay for estimating the 984 links of CU-WART with varying $T h_{\text {Cover }}$. As $T h_{\text {Cover }}$ increases, the delay also increases. Thus, there is a tradeoff between the accuracy and latency in determining $T h_{\text {Cover }}$. From Figure 16, we can estimate how densely the R-tree can be populated and what is the appropriate $T h_{\text {Cover }}$. If $\mathrm{R} / \mathrm{M}=10 \%$ is used, $20 \mathrm{~m}$ can be selected for $T h_{\text {Cover }}$. The average distance between links in the R-tree is about 10 meter if $\mathrm{R} / \mathrm{M}=$ $10 \%$ because the total length covered by the CU-WART is about $1 \mathrm{~km}$.

Figure 17 depicts the impact of $T h_{\text {Save }}$ on the auto-

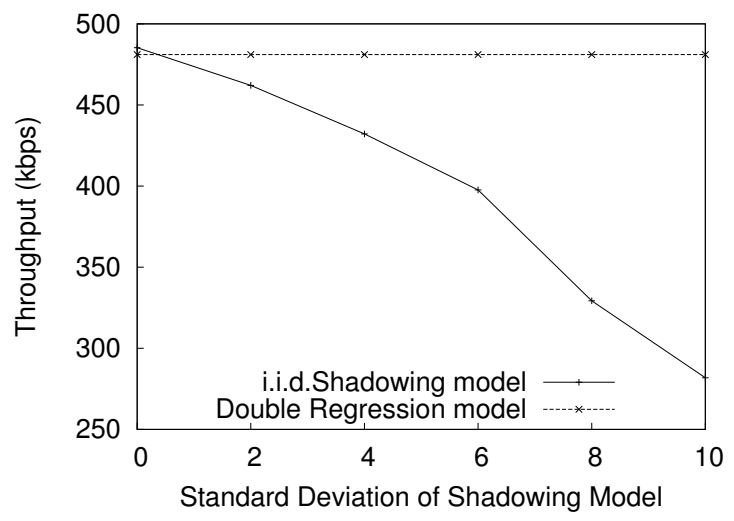

Fig. 12: Impact on Throughput

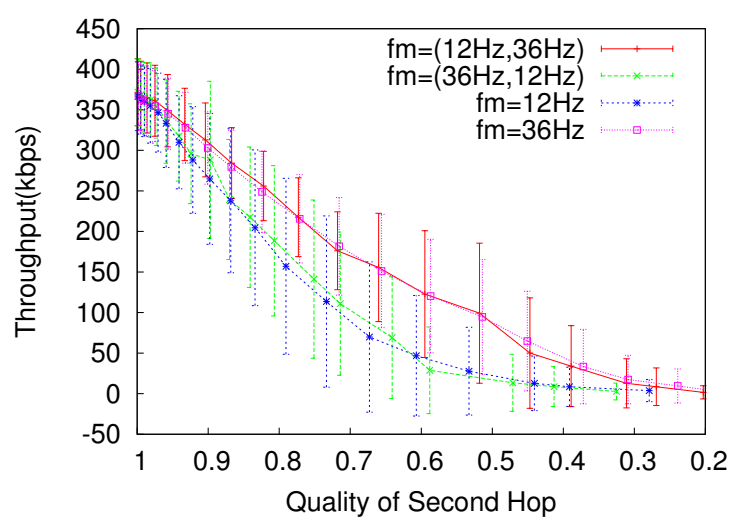

Fig. 14: Impact of PDR on throughput

correlation between two points that are 1 and 5 meter apart when the decorrelation distance is set to 10 . If a small $T h_{\text {Save }}$ is used, more reference links are used to estimate a new link, which results in autocorrelation that is closer to the empirical values. If a large $T h_{\text {Save }}$ is used, less reference links are used and the autocorrelation does not match empirical values. Since the model stores all estimated values that do not have any reference links, a $T h_{\text {Save }}$ value larger than the decorrelation distance does not benefit accuracy.

The average number of the reference links changes as more estimated values are stored in the R-tree. In Figure 18 , the average number of reference links increases as the number of simulation runs increases. With a small $T h_{\text {Save, }}$ estimated results are stored more frequently than when a large $T h_{\text {Save }}$ is used and the number of available reference links increases quickly.

\subsection{Impact on Mobile Links}

To investigate the impact of the model on the throughput of a mobile link, we combine the path loss models being investigated with the Rayleigh model to serve as the multi-path component. In this experiment, we 

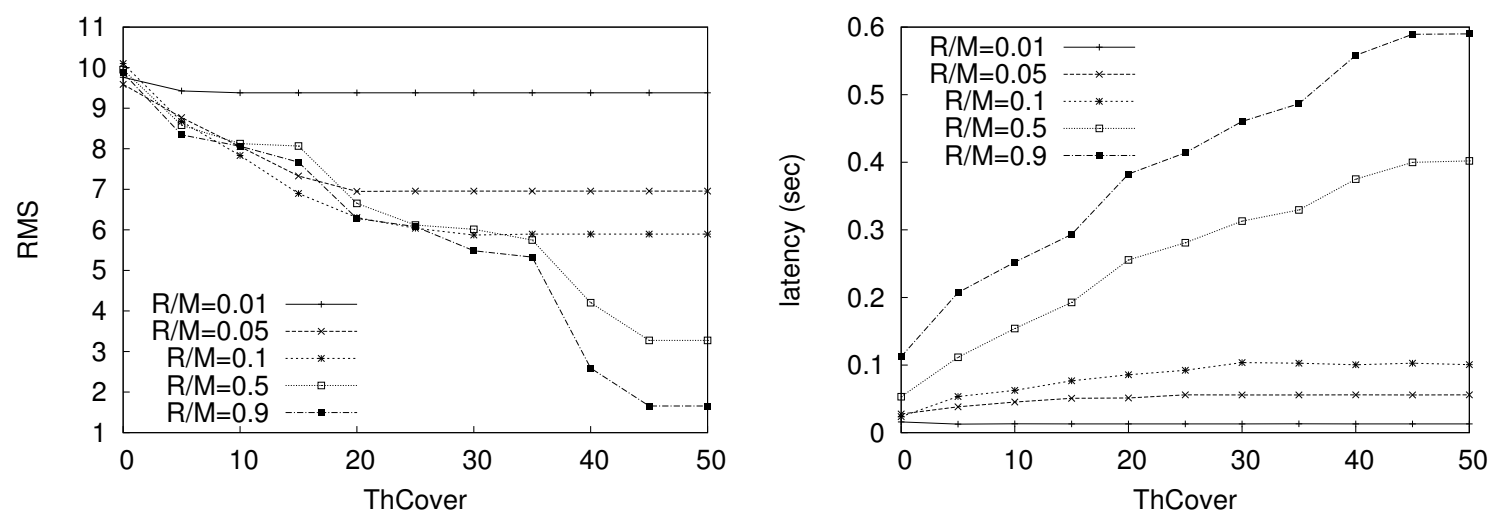

Fig. 15: Impact of $T h_{\text {Cover }}$ on the accuracy of the Fig. 16: Impact of $T h_{\text {Cover }}$ on the performance model

of the model
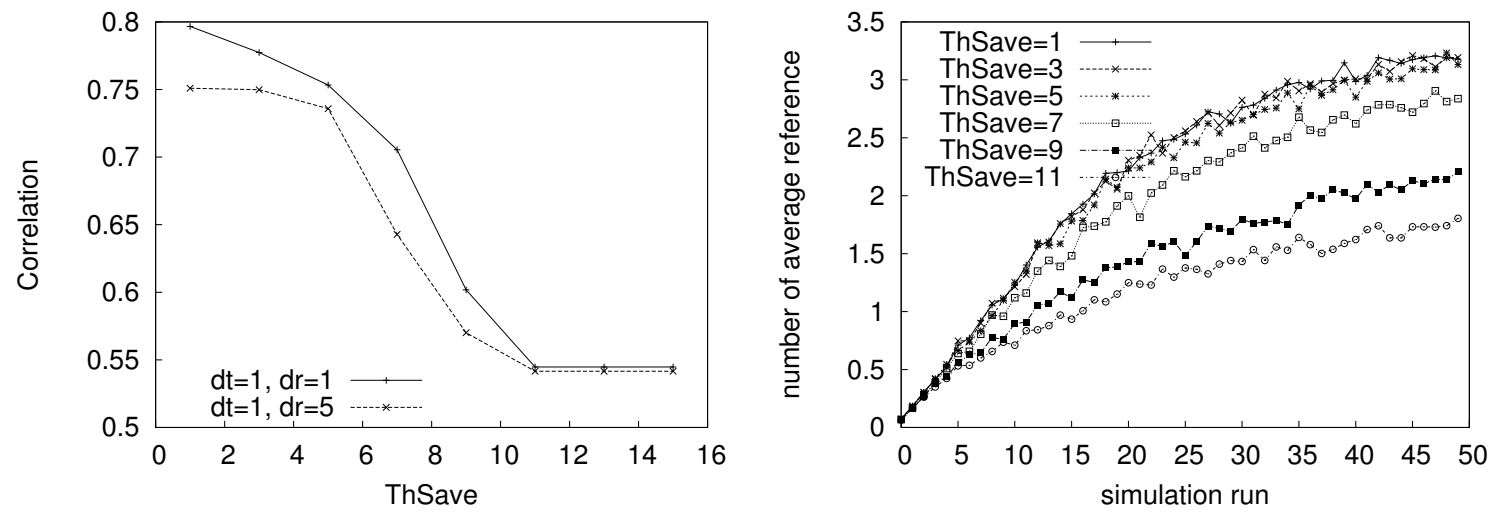

Fig. 17: Impact of $T h_{\text {Save }}$ on the autocorrelation Fig. 18: Impact of simulation run on the average of the model

number of the reference links

use the the CU Wide Area Radio Testbed (CU-WART) map (Figure 3a) and initially place a sender at (303, $98)$ and a receiver at $(250,74)$ in a street. We move the receiver along the street at a speed of $0.1 \mathrm{~m} / \mathrm{s}$ so that there is a line of sight (LOS) between the sender and the receiver, which results in no significant decrease in path loss over distance. The data rate is $11 \mathrm{Mbps}$ and the packet size is 1000 bytes. We use CBR traffic with an interval of $0.2 \mathrm{msec}$ and ftp traffic so that the channel is saturated. Transmission power is $20 \mathrm{dBm}$ and DSR is used for routing.

Figures 19 to 24 show the throughput of CBR and TCP over time when different path loss models are used. When Rayleigh fading with two ray ground model is used, the throughput decreases as the distance between the receiver and the sender increases because the path loss increases. As the path loss increases, periods of no throughput are observed more frequently because the average fade duration (AFD) increases due to fading. Similar throughput decrease is observed in the log normal shadowing model because the average path loss also increases with distance. Because the path loss in the two-ray ground model is a strict function of distance, as the distance increases it experiences increasingly worse channels. While the same is true for log-normal shadowing on average, it benefits sometimes from good quality channels due to the stochastic nature of the log-normal component.

When Rayleigh with DR is used, the throughput is stable because the path loss does not change much when the receiver moves along a road or corridor due to LOS. That means the two ray ground model and the log normal shadowing model do not consider the geographical characteristic and can cause unrealistic throughput loss due to Rayleigh fading even though there are good links due to LOS. The run time overhead of the proposed model is between 2.5 to 3 folds of the two-ray ground model, which is acceptable compared to 100x slowdowns and the detailed GIS models required with ray tracing [27].

\section{Conclusion}

Accurate and efficient channel models are critical for improving the fidelity of wireless network simulation. In this paper, we considered the problem of path loss channel models. Path loss is affected by the RF shadows 


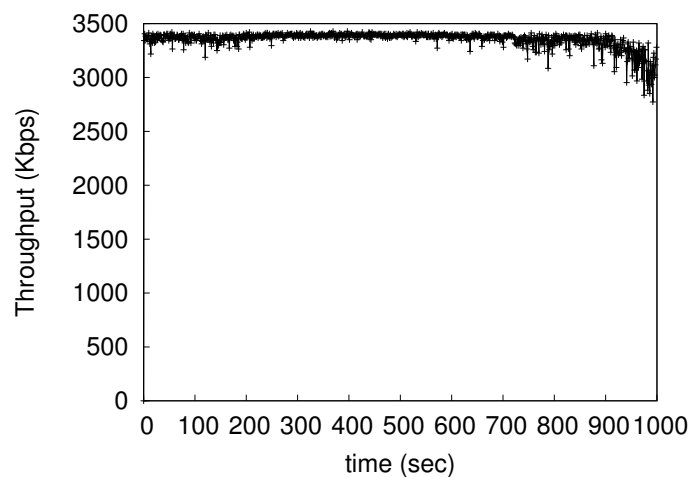

Fig. 19: CBR throughput with DR-Rayleigh model

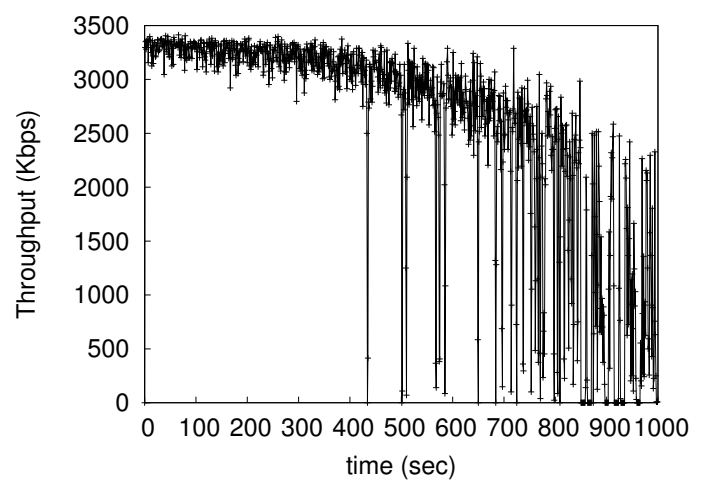

Fig. 20: CBR throughput with Two ray ground + Rayleigh model

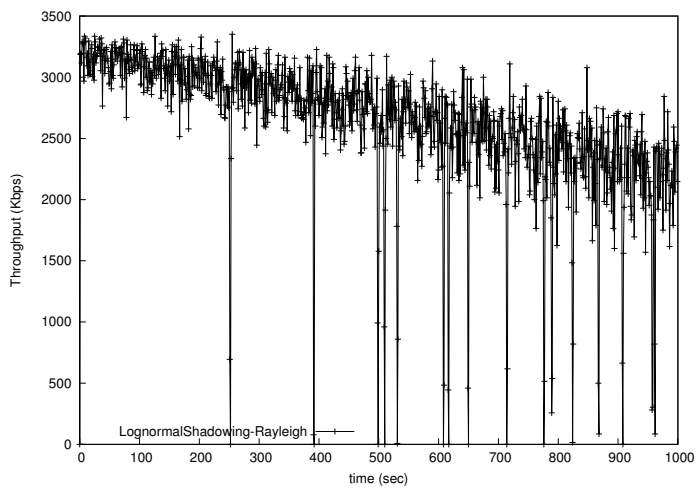

Fig. 21: CBR throughput with Lognormal Shadowing + Rayleigh model

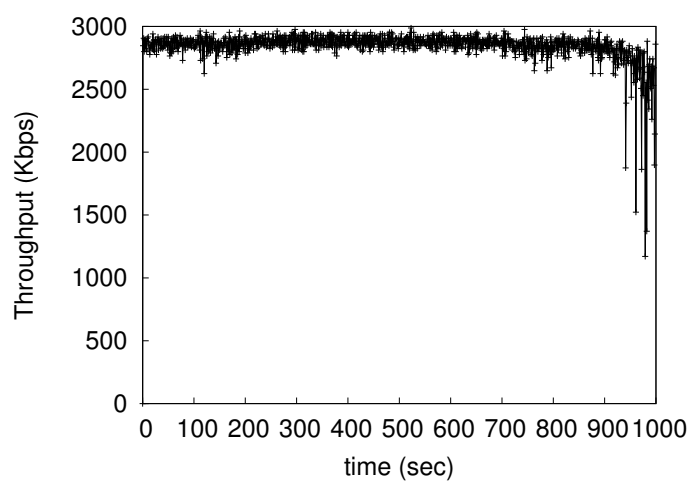

Fig. 22: TCP throughput with DR + Rayleigh model

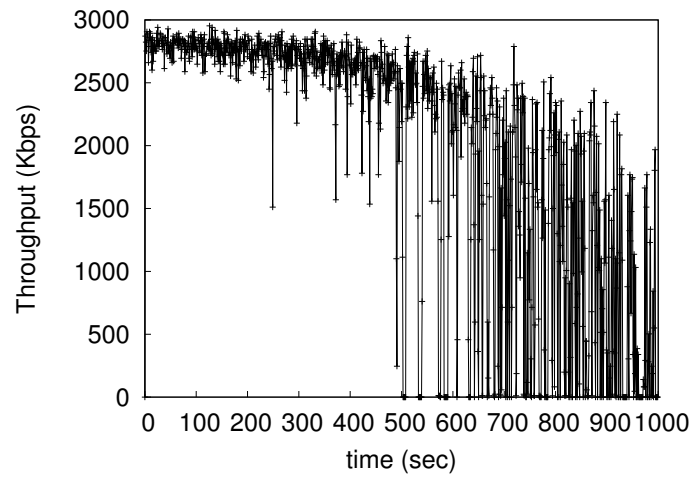

Fig. 23: TCP throughput with Two ray ground + Rayleigh model

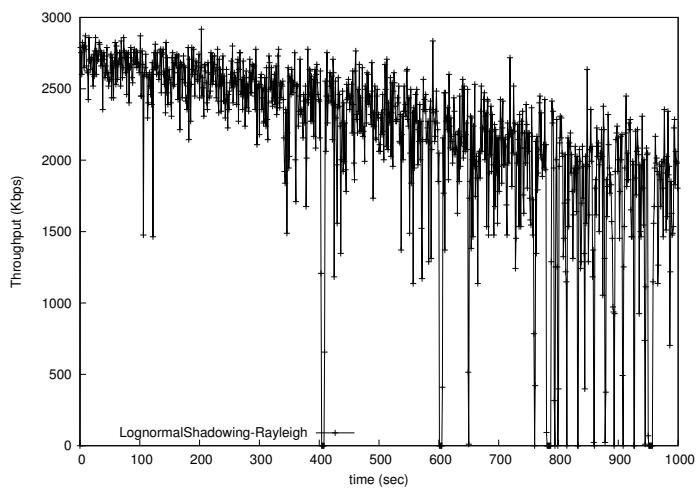

Fig. 24: TCP throughput with Lognormal Shadowing + Rayleigh model

in the environment. Models used in most popular packet simulators are inaccurate because they do not consider the impact of RF shadows on a link such as corridors or building shadows. On the other hand, accurate models can be built using either detailed site-surveys (requiring a prohibitive number of measurement experiments) or ray tracing (which is computationally very difficult and requires precise GIS). Thus, there is a need for accurate and efficient path loss models.

In response to this need, we proposed a new path loss for a geographically accurate, efficient, and flexible path loss model. The key idea of the proposed model is to estimate a plausible value for new channels when both sender and receiver move. It can also be used to substantially reduce the cost of ray tracing and still obtain plausible models of the environment. Both of these applications represent a considerable benefit over the state of the art in this area. DR provides for the first time a spatially-coherent, low-overhead, and site-faithful path loss simulation model.

\section{ACKNOWLEDGEMENTS}

This work was partially supported by grant No. NPRP 08562-1-095 from the Qatar National Research Fund (QNRF), grants CNS-0916323 and CNS-0958501 from the US National Science Foundation, and the IT R\&D program of MSIP/KEIT [10045459]. 


\section{REFERENCES}

[1] D. Kotz, C. Newport, and C. Elliott, "The mistaken axioms of wireless-network research," Dept. of Computer Science, Dartmouth College, Tech. Rep. TR2003-467, July 2003.

[2] L. Perrone and Y. Yuan, "Modeling and simulation best practices for wireless ad hoc networks," in Simulation Conference, 2003. Proceedings of the 2003 Winter, vol. 1, Dec. 2003, pp. 685-693.

[3] J. Liu, Y. Yuan, D. M. Nicol, R. S. Gray, C. C. Newport, D. Kotz, and L. F. Perrone, "Simulation validation using direct execution of wireless ad-hoc routing protocols," in Proc. PADS, 2004.

[4] D. Couto, "A high-throughput path metric for multi-hop wireless routing," in MobiCom, 2004.

[5] J. Bicket, D. Aguayo, S. Biswas, and R. Morris, "Architecture and evaluation of an unplanned 802.11 b mesh network," in MobiCom '05: Proceedings of the 11th annual international conference on Mobile computing and networking, 2005.

[6] A.Adya, P.Bahl, J.Padhye, A.Wolman, and L.Zhou, "A multi-radio unification protocol for ieee 802.11 wireless networks," Microsoft Research, Tech. Rep., Jul. 2003.

[7] R.Draves, "Comparison of routing metrics for static multi-hop wireless networks," in SIGCOMM, 2004.

[8] T. Camp, J. Boleng, and V. Davies, "Mobility models for ad hoc network simulations," Wireless Communication and Mobile Computing (WMCM), 2002, special Issue on Mobile Ad Hoc Networking: Research, Trends and Applications.

[9] J. Yoon, M. Liu, and B. Noble, "Random waypoint considered harmful," in Proc. IEEE Infocom, 2003.

[10] R. Punnoose, P. Nikitin, and D. Dtancil, "Efficient simulation of ricean fading within a packet simulator," in Vehicular Technology Conference - Fall, Sep. 2000.

[11] ISI, "ns: Change history," http://www.isi.edu/nsnam/ns/ CHANGES.html.

[12] T. S. Rappaport, Wireless Communications : Principles and Practice. Prentice Hall, 2002.

[13] M. Gudmundson, "Correlation model for shadow fading in mobile radio systems," in Electron. Lett., vol. 27, Nov. 1991, pp. 21452146.

[14] N. Jalden, P. Zetterberg, B. Ottersten, A. Hong, and R. Thoma "Correlation properties of large scale fading based on indoor measurements," in Wireless Communications and Networking Conference, 2007 WCNC 2007 IEEE, March 2007, pp. 1894-1899.

[15] W. Jakes Jr., Microwave Mobile communications. Wiley, 1974.

[16] S. Zvanovec, P. Pechac, and M. Klepal, "Wireless lan networks design:site survey or propagation modeling?" in Wireless Lan Networks Design, 2003.

[17] D. I. Laurenson, "Indoor radio channel propagation modelling by ray tracing techniques," Ph.D. dissertation, The University of Edinburgh, 1994.

[18] M. Nidd, S. Mann, and J. Black, "Using ray tracing for site-specific indoor radio signal strengthanalysis," in Vehicular Technology Conference, 1997 IEEE 47th, vol. 2, May 1997, pp. 795 - 799.

[19] G. E. Athanasiadou and A. R. Nix, "A novel 3-d indoor raytracing propagation model:the path generator and evaluation of narrow-band and wide-band predictions," in IEEE Transactions on Vehicular Technology, vol. 49, july 2000.

[20] N. Patwari, Y. Wang, and R. O'Dea, “The importance of the multipoint-to-multipoint indoor radio channel in ad hoc networks," 2002. [Online]. Available: citeseer.ist.psu.edu/ patwari02importance.html

[21] Y. Xu and W.-C. Lee, "Exploring spatial correlation for link quality estimation in wireless sensor networks," in PERCOM '06: Proceedings of the Fourth Annual IEEE International Conference on Pervasive Computing and Communications. Washington, DC, USA: IEEE Computer Society, 2006, pp. 200-211.

[22] J.-M. Dricot and P. D. Doncker, "Integrated ad-hoc and cellular networking in indoor or faded environments," in IWCMC '09: Proceedings of the 2009 International Conference on Wireless Communications and Mobile Computing. New York, NY, USA: ACM, 2009, pp. 333-337.

[23] J. Robinson, R. Swaminathan, and E. W. Knightly, "Assessment of urban-scale wireless networks with a small number of measurements," in Proceedings of the 14th ACM international conference on Mobile computing and networking, ser. MobiCom '08. New York, NY, USA: ACM, 2008, pp. 187-198. [Online]. Available: http://doi.acm.org/10.1145/1409944.1409967
[24] A. Konak, "Estimating path loss in wireless local area networks using ordinary kriging," in Winter Simulation Conference (WSC), Proceedings of the 2010, Dec. 2010, pp. 2888 - 2896.

[25] I. Stepanov, D. Herrscher, and K. Rothermel, "On the impact of radio propagation models on manet simulation results," University Stuttgart, Fakultat 5, Germany, Computer Science Archive, 2005.

[26] M. Catedra, J. Perez, F. Saez de Adana, and O. Gutierrez, "Efficient ray-tracing techniques for three-dimensional analyses ofpropagation in mobile communications: application to picocell andmicrocell scenarios," in IEEE Antennas and Propagation Magazine, Apr 1998, pp. 15-28.

[27] J. Dricot and P. Doncker, "High-accuracy physical layer model for wireless network simulations in ns," in International Workshop on Wireless Ad-hoc Networks, 2004. [Online]. Available: citeseer.ist.psu.edu/dricot04highaccuracy.html

[28] V. Sridhara and S. Bohacek, "Realistic propagation simulation of urban mesh networks," Computer Networks, vol. 51, no. 12, 2007.

[29] J. Lu, H. Bertoni, K. Remley, W. Young, and J. Ladbury, "Sitespecific models of the received power for radio communication in urban street canyons," Antennas and Propagation, IEEE Transactions on, vol. 62, no. 4, pp. 2192-2200, April 2014.

[30] S.O.Rice, "Mathematical analysis of random noise," Bell System Technology Journal, vol. 23,24, pp. 282-332,46-156, 1944,1945.

[31] M. Patzold, U. Killat, F. Laue, and Y. Li, "On the statistical properties of deterministic simulation modelsfor mobile fading channels," Vehicular Technology, IEEE Transactions on, vol. 47, pp. 254 - 269, Feb. 1998.

[32] X. Cai and G. B. Giannakis, "A two-dimensional channel simulation model for shadowing processes," IEEE Trans. on Vehicular Technology, November 2003.

[33] A. N. Zhenyu Wang, Eustace K. Tameh, "Simulating correlated shadowing in mobile multihop relay/ad-hoc networks," 2006. [Online]. Available: http://ieee802.org/16

[34] Z. Li, R. Wang, and A. F. Molisch, "Shadowing in urban environments with microcellular or peer-to-peer links," in 6th EUCAP Conference, 2011.

[35] S. S. Szyszkowicz, F. Alaca, H. Yanikomeroglu, and J. S. Thompson, "Aggregate interference distribution from large wireless networks with correlated shadowing: An analyticalnumericalsimulation approach," IEEE Transactions on Vehicular Technology, vol. 6, no. 6, July 2011.

[36] M. Rahnema, UMTS network planning, optimization, and interoperation with GSM. Wiley, 2005.

[37] D. J. Cichon and T. Krner, "Cost231 final report, chapter 4," 1997, http://www.lx.it.pt/cost231/final_report.htm.

[38] J. A. R. Azevedo and F. Santos, "An empirical propagation model for forest environments at tree trunk level," IEEE Trans. Antennas Propag., vol. 59, no. 6, p. 23572367, 2011.

[39] I. StatSoft, “General regression models (grm)," Website, 1984, http: / /www.statsoft.com/textbook/stgrm.html.

[40] N. Beckmann, H.-P. Kriegel, R. Schneider, and B. Seeger, "The $\mathrm{r}^{*}$-tree: an efficient and robust access method for points and rectangles," in International Conference on Management of Data. ACM, 1990, pp. 322-331.

[41] A. Gehring, M. Steinbauer, I. Gaspard, and M. Grigat, "Empirical channel stationarity in urban environments," in EPMCC, 2001.

[42] G. Gaertner and V. Cahill, "Understanding link quality in 802.11 mobile ad hoc networks," IEEE Internet Computing, vol. 8, no. 1, 2004.

[43] Wildpackets, "Converting signal strength percentage to $\mathrm{dbm}$ values," 2002. [Online]. Available: http://iutsa.unice.fr/ frati/ Wireless/TD/Etude\%20-\%20Converting_Signal_Strength.pdf

[44] C. Phillips and E. W. Anderson, "CRAWDAD trace cu/cu_wart/2010/path_loss (v. 2011-10-24)," http://crawdad .cs.dartmouth.edu/cu/cu_wart/2010/path_loss, Oct. 2011.

[45] J. Broch, D. Johnson, and D. Maltz, "The dynamic source routing protocol for mobile ad hoc networks," Internet Engineering Task Force, Internet Draft, Jun. 1999, work in progress. 